\title{
Perturbation theory and locality in the field-antifield formalism
}

\author{
Joaquim Gomis ${ }^{\text {a) }}$ \\ Uji Research Center, Yukawa Institute for Theoretical Physics, Kyoto University, \\ Uji 611, Japan \\ Jordi París ${ }^{\text {b) }}$ \\ Departament d'Estructura i Constituents de la Matèria, Universitat de Barcelona, \\ Diagonal 647 E-08028 Barcelona, Catalonia, Spain
}

(Received 1 October 1992; accepted for publication 4 January 1993)

The Batalin-Vilkovisky formalism is studied in the framework of perturbation theory by analyzing the antibracket Becchi-Rouet-Stora-Tyutin (BRST) cohomology of the proper solution $S_{0}$. It is concluded that the recursive equations for the complete proper solution $S$ can be solved at any order of perturbation theory. If certain conditions on the classical action and on the gauge generators are imposed the solution can be taken local.

\section{INTRODUCTION}

The construction of the effective action is a key ingredient in the covariant quantization of gauge theories. One of the most powerful methods to get the effective action for such types of theories is the procedure based in the Becchi-Rouet-Stora-Tyutin (BRST) symmetry, ${ }^{1}$ in particular, its field-antifield formulation. ${ }^{2}$ In this approach the main open questions are the unitarity of the resulting theory and, related to this point, the locality of the gauge fixed action.

An alternative procedure to construct the effective action in the framework of perturbation theory was proposed by Frolov and Slavnov. ${ }^{3}$ They studied the condition imposed by the commutativity of the $S$-matrix operator $\hat{S}$ and the free BRST charge and found a set of recurrent equations for the effective action. In a recent paper ${ }^{4}$ we developed the field-antifield formalism in this framework and showed that it produces a gauge fixed action which coincides with the one obtained by them. The equivalence of both procedures was proved by making two assumptions: (i) there exist suitable power series expansions in the coupling constant for the structure functions and (ii) the existence of solutions of the recurrent equations for the proper solution of the master equation at any order in perturbation theory. In particular, the first assumption allowed us to solve explicitly the lowest order recurrent equations and to show that their solutions coincide with those given in Ref. 3. The problem of unitarity was also considered. Under certain assumptions on the gauge generators, we proved the absence of pairs of singlets ${ }^{5-7}$ and, consequently, that unitarity ultimately relies on special features of the theory, as for example, the presence or absence of classical gauge invariant degrees of freedom with negative norm.

In this paper we will analyze the antibracket BRST cohomology defined in the space of all functionals (i.e., local and nonlocal ones) and, in particular, the cohomology associated with the free part of the proper solution $S_{0}$, using homological techniques. By introducing a nilpotent operator $\sigma$, we will construct an operator that counts the number of fields and antifields and show that the antibracket BRST cohomology of $S$ is isomorphic to the kcrnel of this operator. (The idea of relating the BRST cohomology with the kernel of a suitable number operator has been widely used in the literature. See, for example, Refs. 8-10.) This study allows

\footnotetext{
${ }^{2}$ Permanent address: Departament d'Estructura i Constituents de la Matèria, Universitat de Barcelona. Electronic address: Quim@EBUBECM1.bitnet.

${ }^{b)}$ Electronic address: Paris @ EBUBECM1.bitnet.
} 
us to verify the triviality of the cohomology associated with the free part of the proper solution $S_{0}$ except at ghost number 0 and, as a consequence, (i) that the recurrent equations for $S$ can be solved at any order in perturbation theory (The proof of the existence theorem of an effective action in the field-antifield formalism was given earlier in Refs. 11-13) and (ii) that the structure functions have suitable power series expansions in the coupling constant.

On the other hand, the question of the locality of the gauge fixed action in the field-antifield formalism has recently been considered by Henneaux, ${ }^{14}$ studying the local cohomology of the so-called Koszul-Tate differential, and also in Ref. 15. In this paper we also address this problem in the framework of perturbation theory. Assuming the locality of the classical action $S_{\mathrm{cl}}$ and of the generators of the gauge algebra $R_{s \alpha_{s}}^{\alpha_{s-1}}, s=0, \ldots, L$, and the local completeness of the free part of these generators, we shall show that the structure functions defining the gauge structure of the theory and, as a result, the proper solution of the master equation, can be taken to be local.

We have organized the paper as follows: in Sec. II we analyze in detail the antibracket BRST cohomology through the introduction of a contracting homotopy $\sigma$. In Sec. III we provide an alternative proof of the existence theorem of a solution of the master equation for a generic gauge theory, in the framework of perturbation theory. The question of the locality of the proper solution is addressed in Sec. IV and in Sec. V we give some conclusions. We end with two appendices. The first one is devoted to analyzing the questions of the properness and the classical limit of the solution of the master equation in the framework of perturbation theory. Finally, in the second appendix, we justify the form of the structure functions used to derive the above results.

\section{ANTIBRACKET BRST COHOMOLOGY}

In order to analyze the antibracket BRST cohomology defined in the space of all functionals (local and nonlocal ones) for a generic proper solution $S$, it would be useful to recall the so-called regularity conditions ${ }^{12,16}$ and the concept of observable. ${ }^{16}$ Let $I$ be the infinite dimensional space of all historics and $G_{i}=\partial S_{\mathrm{cl}} / \partial \phi^{i}$ the classical equations of motion. Then, the regularity assumption states that $G_{i}$ provide a regular representation of the stationary surface $\Sigma$, where the equations of motion hold. This means that the functions $G_{i}$ can be locally split as $G_{i}=\left(G_{A_{-1}}, G_{a_{-1}}\right)$ in such a way that

(i) $G_{a_{-1}}=0$ is a consequence of $G_{A_{-1}}=0$, and

(ii) the rank of $d G_{A_{-1}}$ is maximal on the stationary surface $\Sigma$.

If for a given theory the regularity conditions are verified, any smooth function vanishing on the stationary surface can be expressed as $\lambda^{A}{ }_{-1} G_{A_{-1}}$, with $\lambda^{A}-1$ smooth functions. Throughout this paper, we will consider classical actions $S_{\mathrm{cl}}$ for which the regularity conditions hold.

With respect to the dynamical observables, following Ref. 16, these are described as smooth functions defined on the stationary surface $\Sigma$. The definition of observable can be extended to the space of all possible histories, $I$, by identifying two functions which coincide on shell, i.e.,

$$
F(\phi) \sim F^{\prime}(\phi) \Leftrightarrow F-F^{\prime}=\lambda^{i} G_{i}
$$

Moreover, in the case of gauge theories, one must further impose that the observables be gauge invariant, at least on the stationary surface, that is

$$
\frac{\partial F}{\partial \phi^{i}} R_{0 \alpha_{0}}^{i}=\lambda^{i} G_{i},
$$

being $R_{0 \alpha_{0}}^{i}$ the generators of the gauge transformations. 
Given that functions defined on $I$ form an algebra, $C(I)$, and that functions which vanish on shell form an ideal $\mathscr{N}$ of this algebra, the algebra of functions defined on the stationary surface $\Sigma$ is isomorphic with the quotient $C(I) / \mathscr{N}$. In presence of gauge invariance, however, this is not the complete story. As it is well known, the gauge transformations are integrable on $\Sigma$ and generate the so-called gauge orbits. Since the gauge invariant functions are constant along the gauge orbits, they induce well defined functions in the quotient space $\Sigma / G, G$ being the gauge orbits. Therefore, the space of observables can be identified in this case with the set of smooth functions on $\Sigma / G, C(\Sigma / G)$.

A cohomological interpretation of this construction was given in Ref. 16. It was found that the set of physically inequivalent observables is isomorphic with the cohomology at ghost number zero of a nilpotent operator $\delta, H_{0}(\delta)$, induced for the proper solution of the master equation through the antibracket, and defined as

$$
\delta F \equiv(-1)^{F}(F, S), \quad H_{0}(\delta)=\left(\frac{\operatorname{Ker} \delta}{\operatorname{Im} \delta}\right)
$$

where the $F$ in the exponent stands for the Grassmann parity of the function $F$.

In what follows, we will try to relate locally the antibracket BRST cohomology of a generic proper solution $S$ and, in particular, the set of physically inequivalent observables, with the kernel of a certain number operator $N$. As a preliminary step, we will perform this construction for a regular action without gauge degrees of freedom.

\section{A. Nongauge theory}

Consider a regular action $S_{\mathrm{cl}}\left(\phi^{i}\right)$ without gauge degrees of freedom and introduce an antifield $\phi_{i}^{*}$ for each classical field $\phi^{i}$, with opposite statistics. (For simplicity, throughout this paper we will assume that the classical fields $\phi^{i}$ obey Bose statistics.) When no gauge degrees of freedom are present, the proper solution is nothing but the classical action $S_{\mathrm{cl}}\left(\phi^{i}\right)$ and the BRST operator $\delta$ is defined as

$$
\delta \phi^{i}=0, \quad \delta \phi_{i}^{*}=\frac{\partial S_{\mathrm{cl}}}{\partial \phi^{i}}=G_{i}(\phi)
$$

Introduce in the space of all histories $I$ the change of coordinates

$$
\phi^{i} \rightarrow \phi_{i}^{\prime} \equiv G_{i}(\phi)
$$

Due to the fact that the action $S_{\mathrm{cl}}$ defines a regular theory without gauge degrees of freedom, the Jacobian matrix of this change

$$
H_{i j}=\frac{\partial \phi_{i}^{\prime}}{\partial \phi^{j}}=\frac{\partial^{2} S_{\mathrm{cl}}}{\partial \phi^{i} \partial \phi^{j}}
$$

does not have null vectors around the stationary surface $G_{i}=0$ (which in the regular case reduces to a point) and, as a consequence, the above change is invertible, at least in a neighborhood of the stationary point. In this neighborhood, the BRST operator $\delta$ acts on the local coordinates as

$$
\delta G_{i}=0, \quad \delta \phi_{i}^{*}=G_{i}
$$

Define locally in the space of fields and antifields an operator $\sigma$ which decreases the ghost number by one unit 


$$
\begin{gathered}
\sigma \phi^{i}=\left(H^{-1}\right)^{i j} \phi_{j}^{*}, \quad \sigma \phi_{i}^{*}=0, \\
\sigma F\left(\phi, \phi^{*}\right)=\sigma \phi^{i} \frac{\partial_{l} F}{\partial \phi^{i}}+\sigma \phi_{i}^{*} \frac{\partial_{l} F}{\partial \phi_{i}^{*}}
\end{gathered}
$$

where $\left(H^{-1}\right)^{i j}$ is the inverse matrix of the Jacobian matrix (2.2). This operator results to be an antiderivation

$$
\sigma(A B)=\sigma A \cdot B+(-1)^{A} A \cdot \sigma B
$$

It is clear that, in general, $\sigma$ will only be defined in a neighborhood of the stationary point, where the change (2.1) and the Jacobian matrix (2.2) are invertible. In terms of the local coordinates $\left(G_{i}, \phi_{i}^{*}\right)$, the action of $\sigma$ reads

$$
\sigma G_{i}=\phi_{i}^{*}, \quad \sigma \phi_{i}^{*}=0
$$

Now, with the operators $\delta$ and $\sigma$ at hand, one can construct a new operator as the following quadratic combination

$$
N=\sigma \delta+\delta \sigma
$$

Its actuation on the local coordinates $\left(G_{i}, \phi_{i}^{*}\right)$ is

$$
N G_{i}=(\sigma \delta+\delta \sigma) G_{i}=\delta \phi_{i}^{*}=G_{i}, \quad N \phi_{i}^{*}=(\sigma \delta+\delta \sigma) \phi_{i}^{*}=\sigma G_{i}=\phi_{i}^{*} .
$$

Furthermore, from the definition of $\delta$, Eq. (2.3), and $\sigma$, Eq. (2.4), $N$ is a derivation

$$
\begin{gathered}
N F\left(\phi, \phi^{*}\right)=N G_{i} \frac{\partial_{l} F}{\partial G_{i}}+N \phi_{i}^{*} \frac{\partial_{l} F}{\partial \phi_{i}^{*}}, \\
N(A B)=N A \cdot B+A \cdot N B
\end{gathered}
$$

and, as a consequence of the above relations, it counts the number of equations of motion $G_{i}$ and of the antifields $\phi_{i}^{*}$, i.e.,

$$
N A=N\left(G_{i_{1}} \cdots G_{i_{n}} A^{i_{1} \cdots i_{n}, j_{1} \cdots j_{m}} \phi_{j_{1}}^{*} \cdots \phi_{j_{m}}^{*}\right)=(n+m) A .
$$

In this sense, the operator $\sigma(2.4)$ is a contracting homotopy.

Consider now a smooth function $F(\phi)$ defined in the space of all histories $I$. The assumed regularity conditions allow us to write $F$ as

$$
F(\phi)=F\left(\phi_{0}\right)+\lambda^{i}(\phi) G_{i}(\phi),
$$

where $F\left(\phi_{0}\right)$ is the value of $F$ at the stationary point and $\lambda^{i}$ are suitable smooth coefficients. For the same reason, these coefficients can be expanded in the same way

$$
\lambda^{i}(\phi)=\lambda^{i}\left(\phi_{0}\right)+\lambda^{i j}(\phi) G_{j},
$$

and applying this procedure recursively we arrive at

$$
F(\phi)=F\left(\phi_{0}\right)+\sum_{n} \lambda^{i_{1} \cdots i_{n}} G_{i_{1}} \cdots G_{i_{n}},
$$


where we must understand that this expansion is valid in a neighborhood of the stationary point. If instead of functions $F(\phi)$, we consider functions $F\left(\phi, \phi^{*}\right)$, defined in the space of fields and antifields

$$
F\left(\phi, \phi^{*}\right)=F(\phi)+\sum_{n>0} F^{i_{1} \cdots i_{n}}(\phi) \phi_{i_{1}}^{*} \cdots \phi_{i_{n}}^{*},
$$

with $F(\phi), F^{i_{1} \cdots i_{n}}(\phi)$ smooth functions of the fields $\phi^{i}$, the result (2.5) leads us to the expansion around the stationary point

$$
F\left(\phi, \phi^{*}\right)=F\left(\phi_{0}\right)+\sum_{n, m} G_{i_{1}} \cdots G_{i_{n}} F^{i_{1} \cdots i_{n}, j_{1} \cdots j_{m}} \phi_{j_{1}}^{*} \cdots \phi_{j_{m}}^{*}
$$

Let us see now how to realize the inequivalent observables in a regular theory. From the definition of observable it is clear that all the elements belonging to a given equivalence class are characterized for having the same value in the stationary point. Then, the most natural representative in each equivalence class is a constant function over all the space of histories $I$. Therefore,

$$
C(I) / \mathscr{N} \simeq\left\{F(\phi) / F(\phi)=F\left(\phi_{0}\right)=\text { ct. }\right\} \simeq\{\text { observables }\}
$$

On the other hand, consider the action of the operator $N$ on a generic function $F\left(\phi, \phi^{*}\right)$ of the form (2.6)

$$
N F\left(\phi, \phi^{*}\right)=0+\sum_{n, m}\left[(n+m) G_{i_{1}} \cdots G_{i_{n}} F^{i_{1} \cdots i_{n}, j_{1} \cdots j_{m}} \phi_{j_{1}}^{*} \cdots \phi_{j_{m}}^{*}\right]
$$

It is clear that the kernel of this operator is precisely

$$
\operatorname{ker} N=\left\{F\left(\phi, \phi^{*}\right) / F=F\left(\phi_{0}\right)=\text { ct. }\right\},
$$

which is nothing but the set of inequivalent observables (2.7). In summary, by constructing an operator $N$ which counts the number of equations of motion and of antifields, we have characterized locally the set of physically inequivalent observables as the kernel of this operator.

\section{B. Lth stage reducible gauge theory}

Let us see now how we can implement the constructions of $\sigma$ and $N$ to the case of a general $L$ th stage reducible gauge theory. To perform this program it will be useful to note the so-called abelianization theorem. ${ }^{11,16,17}$ This theorem states that, if the regularity conditions are verified, there always exist locally smooth coordinates $\phi^{i}=\left(\phi^{A}-1, \phi^{a}-1\right)$ in which the equations of motion and the reducibility functionals $R_{s \alpha_{s}}^{\alpha_{s-1}}$ take the simple form,

$$
\begin{gathered}
G_{i}=\left(G_{A_{-1}}, G_{a_{-1}} \equiv\right) \\
R_{s \alpha_{s}}^{\alpha_{s-1}}=\left(\begin{array}{cc}
R_{A_{s}}^{A_{s-1}}=0 & R_{A_{s-1}}^{a_{s-1}}=\delta_{A_{s}}^{a_{s-1}} \\
R_{a_{s}}^{A_{s-1}=0} & R_{a_{s}}^{a_{s-1}}=0
\end{array}\right), \quad s=0, \ldots, L-1, \\
\left.R_{L \alpha_{L}}^{\alpha_{L-1}=\left(R_{\alpha_{L}}^{A_{L-1}}=0,\right.} \quad R_{\alpha_{L}}^{a_{L-1}}=\delta_{\alpha_{L}}^{a_{L-1}}\right),
\end{gathered}
$$

where the indices $a_{s-1}, A_{s}$ range over the same values. 
The particular form of the generators of the gauge transformations in these coordinates shows that the fields $\phi^{A}-1$ are the independent gauge invariant degrees of freedom, while $\phi^{a}-1$ are "pure gauge." It should be stressed also that, in general, the separation (2.8) cannot be done globally with a single set of independent equations of motion $G_{A_{-1}}$.

All these considerations imply that in the space of fields and antifields there always exist locally smooth coordinates

$$
C_{k}^{\prime \alpha_{k}} \equiv\left(C_{k}^{A_{k}}, C_{k}^{a_{k}}\right), \quad C_{k \alpha_{k}}^{* *} \equiv\left(C_{k A_{k}}^{*}, C_{k a_{k}}^{*}\right), \quad k=-1,0, \ldots, L
$$

where we have defined $C_{-1}^{\prime \alpha} \equiv \phi^{\prime i}, C_{-1, \alpha_{-1}}^{\prime *} \equiv \phi_{i}^{\prime *}$, in which locally the proper solution of the master equation takes the form

$$
S=S_{\mathrm{cl}}\left(\phi^{A-1}\right)+\sum_{s=0}^{L} C_{s-1, a_{s-1}}^{*} \delta_{A_{s}}^{a_{s-1}} C_{s}^{A_{s}} .
$$

Therefore, the BRST operator $\delta$ associated with $S$ is expressed in terms of these variables as

$$
\begin{aligned}
& \delta \phi^{A}-1=0, \\
& \delta C_{0}^{A_{0}}=0, \quad \delta \phi^{a-1}=\delta_{A_{0}}^{a_{-1}} C_{0}^{A_{0}}, \\
& \vdots \\
& \delta C_{k}^{A_{k}}=0, \quad \delta C_{k-1}^{a_{k-1}}=(-1)^{k} \delta_{A_{k}}^{a_{k-1}} C_{k}^{d_{k}}, \\
& \delta C_{L}^{\alpha_{L}}=0, \quad \delta C_{L-1}^{\alpha_{L-1}}=(-1)^{L} \delta_{\alpha_{L}}^{a_{L-1}} C_{L}^{\alpha_{L}},
\end{aligned}
$$

while for the antifields we have

$$
\begin{gathered}
\delta \phi_{A_{-1}}^{*}=\frac{\partial S_{\mathrm{cl}}}{\partial \phi^{A_{-1}}}=G_{A_{-1}}\left(\phi^{\left.B_{-1}\right)}\right. \\
\delta C_{0 A_{0}}^{*}=\phi_{a_{-1}}^{*} \delta_{A_{0}}^{a}, \quad \delta \phi_{a_{-1}}^{*}=0 \\
\vdots \\
\delta C_{k A_{k}}^{*}=C_{k-1, a_{k-1}}^{*} \delta_{A_{k}}^{a_{k-1}}, \quad \delta C_{k-1, a_{k-1}}^{*}=0 \\
\vdots \\
\delta C_{L \alpha_{L}}^{*}=C_{L-1, a_{L-1}}^{*} \delta_{\alpha_{L}}^{a_{L-1}}, \quad \delta C_{L-1, a_{L-1}}^{*}=0
\end{gathered}
$$

Note that, except for the classical gauge invariant degrees of freedom $\phi^{A}-1$ and their antifields, all the other fields are organized in cohomological trivial pairs.

Now, let us construct the contracting homotopy $\sigma$, in such a way that the operator $N=\delta \sigma+\sigma \delta$ acts as a number operator. As in the precedent subsection, consider the coordinate change 


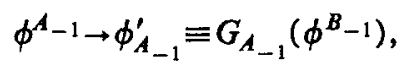

which will be invertible locally due to the fact that the functions $G_{A_{-1}}$ have been chosen to be independent. Therefore, the Jacobian matrix

$$
H_{A_{-1}, B_{-1}}=\frac{\partial \phi_{A_{-1}}^{\prime}}{\partial \phi^{B}-1}=\frac{\partial^{2} S_{\mathrm{cl}}}{\partial \phi^{A_{-1}} \partial \phi^{B_{-1}}},
$$

turns out to be invertible locally. In such conditions, it is easy to see that the contracting homotopy $\sigma$ which realizes the above program acts on the fields as

$$
\begin{aligned}
& o G_{A_{-1}}=\phi_{A_{-1}}^{*}, \\
& \sigma C_{0}^{A_{0}}=\delta_{a_{-1}}^{A_{0}} \phi^{a_{-1}}, \quad \sigma \phi^{a_{-1}}=0, \\
& \sigma C_{k}^{A_{k}}=(-1)^{k} \delta_{a_{k-1}}^{A_{k}} C_{k-1}^{a_{k-1}}, \quad \sigma C_{k-1}^{a_{k-1}}=0, \\
& \sigma C_{L}^{\alpha_{L}}=(-1)^{L} \delta_{a_{L-1}}^{\alpha_{L}} C_{L-1}^{a_{L-1}}, \quad \sigma C_{L-1}^{a_{L-1}}=0
\end{aligned}
$$

and on the antifields

$$
\begin{gathered}
\sigma \phi_{A_{-1}}^{*}=0, \\
\sigma C_{0 A_{0}}^{*}=0, \quad \sigma \phi_{a_{-1}}^{*}=C_{0 A_{0}}^{*} \delta_{a_{-1}}^{A_{0}}, \\
\vdots \\
\sigma C_{k A_{k}}^{*}=0, \quad \sigma C_{k-1, a_{k-1}}^{*}=C_{k A_{k}}^{*} \delta_{a_{k-1}}^{\alpha_{k}}, \\
\vdots \\
\sigma C_{L \alpha_{L}}^{*}=0, \quad \sigma C_{L-1, a_{L-1}}^{*}=C_{L \alpha_{L}}^{*} \delta_{a_{L-1}}^{\alpha_{L}},
\end{gathered}
$$

being as in the regular case nilpotent, $\sigma^{2}=0$, and decreasing the ghost number by one unit.

With these definitions of $\delta$ and $\sigma$ one can see that the operator $N$ acts on the above coordinates as

$$
N C_{k}^{\prime \alpha_{k}}=C_{k}^{\prime \alpha_{k}}, \quad N C_{k \alpha_{k}}^{\prime *}=C_{k \alpha_{k}}^{\prime *}
$$

and counts the number of independent equations of motion $G_{A_{-1}}$, fields $\phi^{a-1}$, ghosts, ..., and the corresponding antifields. Here and from now on we define $C_{-1}^{\prime A_{-1}} \equiv G_{A_{-1}}, C_{-1, A_{-1}}^{*} \equiv \phi_{A_{-1}}^{*}$.

Now, consider a smooth function of the classical fields $F(\phi)$. Assuming the regularity conditions we can expand it in a given local chart as 


$$
\begin{aligned}
F(\phi) & =\widetilde{F}\left(G_{A_{-1}}, \phi^{a_{-1}}\right) \\
& =\widetilde{F}\left(0, \phi^{a_{-1}}\right)+\lambda^{A_{-1}}(\phi) G_{A_{-1}} \\
& =\widetilde{F}(0,0)+\sum G_{A_{-1}} \cdots G_{D_{-1}} \widetilde{F}_{a_{-1} \cdots d_{-1}}^{A_{1} \cdots D_{-1}} \phi^{a_{-1} \cdots \phi^{d_{-1}}}
\end{aligned}
$$

Applying the same argument, a smooth function of the fields and antifields $F\left(C_{s}, C_{s}^{*}\right)$ of the type

$$
F\left(C_{s}, C_{s}^{*}\right)=F(\phi)+\sum_{n, m ; s_{i}>-1} C_{s_{1}}^{\alpha_{s_{1}}} \cdots C_{s_{n}}^{\alpha_{s_{n}}} F_{\alpha_{s_{1}} \cdots \alpha_{s_{n}}}^{\beta_{t_{1}} \cdots \beta_{t_{m}}}(\phi) C_{t_{1} \beta_{t_{1}}}^{*} \cdots C_{t_{m} \beta_{t_{m}}^{*}}^{*}
$$

with $F(\phi), F_{\alpha_{s_{1}} \cdots \alpha_{s_{n}}}^{\beta_{t_{1}} \cdots \beta_{t_{m}}}(\phi)$ smooth functions, admits an expansion in terms of the coordinates $\left(C_{s}^{\prime}, C_{s}^{\prime *}\right)$ of the following form:

$$
\begin{aligned}
F\left(C_{s}, C_{s}^{*}\right) & =\tilde{F}\left(C_{s}^{\prime}, C_{s}^{\prime *}\right) \\
& =\tilde{F}(0,0)+\sum_{n, m} C_{s_{1}}^{\prime \alpha_{s_{1}}} \cdots C_{s_{n}}^{\prime \alpha_{s_{n}}} \widetilde{F}_{\alpha_{s_{1}} \cdots \alpha_{s_{n}} \beta_{t_{1}} \cdots \beta_{t_{m}}} C_{t_{1} \beta_{t_{1}}}^{\prime *} \cdots C_{t_{m} \beta_{t_{m}}}^{* *} \\
& =\widetilde{F}(0,0)+\sum_{n, m} \widetilde{F}_{n, m}\left(C_{s}^{\prime}, C_{s}^{\prime *}\right)
\end{aligned}
$$

where $\tilde{F}(0,0)$ is the value of $F(\phi)$ at the point $G_{A_{-1}}=\phi^{a-1}=0$.

Let us consider the gauge invariant functions. We have

$$
\frac{\partial F(\phi)}{\partial \phi^{i}} R_{0 \alpha_{0}}^{i}=\left.\lambda^{i} G_{i} \Rightarrow \frac{\partial \widetilde{F}}{\partial \phi^{a}-1}\right|_{G_{A_{-1}}=0}=\left.0 \Rightarrow \widetilde{F}\left(G_{A_{-1}}, \phi^{a}-1\right)\right|_{G_{A_{-1}}=0}=\widetilde{F}(0,0),
$$

that is, on the stationary surface, described locally by the coordinates $\phi^{a}-1$, the gauge invariant function $F$ is a constant. On the other hand, two gauge invariant observables $F(\phi), F^{\prime}(\phi)$ are equivalent if their constant value on the stationary surface is the same. Therefore, we obtain a similar result as that we arrived in the regular case, namely, in each class of equivalent observables we can choose a representative which is a constant function over all the space of histories $I$. Moreover, taking into account the expansion (2.12) and the actuation of $N$, we finally conclude that the kernel of this operator, i.e., the set of constant functions, is isomorphic to the set of equivalence classes of observables, or $H_{0}(\delta)$

$$
H_{0}(\delta) \simeq\{\text { observables }\} \simeq \operatorname{ker} N=\left\{F\left(C_{s}, C_{s}^{*}\right) / F=\widetilde{F}(0,0)=\text { ct. }\right\}
$$

The construction of the contracting homotopy $\sigma$ and of the operator $N$ allows us to gain a better understanding of the antibracket BRST cohomology of a generic proper solution $S$. As it is well known, given a proper solution $S$, one defines the nilpotent BRST operator $\delta$ and its corresponding cohomology spaces as 


$$
\delta F=(-1)^{F}(F, S), \quad H_{k}(\delta)=\left(\frac{\operatorname{Ker} \delta}{\operatorname{Im} \delta}\right)_{k}
$$

being $k$ the grading of the corresponding space, i.e., the ghost number.

Until now we have been studying the cohomology space $H_{0}(\delta)$, which gives the physically inequivalent observables. To study the case $k \neq 0$ consider a smooth function $F\left(C_{s}, C_{s}^{*}\right)$ such that gh $F=k \neq 0$ and $\delta F=0$. Given that gh $F \neq 0$, we have an expansion of the type (2.12) in terms of the local coordinates $\left(C_{s}^{\prime}, C_{s}^{*}\right)$, with no constant term $\widetilde{F}(0,0)$. If we apply now the operator $N$ to a homogeneous term of the expansion of $F, \widetilde{F}_{n, m}$, which also verifies $\delta \widetilde{F}_{n, m}=0$, we have, on the one hand

$$
N \tilde{F}_{n, m}=N\left(C_{s_{1}}^{\alpha \alpha_{s_{1}} \cdots C_{s_{n}}^{\prime \alpha_{s_{n}}}} \tilde{F}_{\alpha_{s_{1}} \cdots \alpha_{s_{n}}}^{\beta_{t_{1}} \cdots \beta_{t_{m}}} C_{t_{1} \beta_{t_{1}}}^{\prime *} \cdots C_{t_{m} \beta_{t_{m}}}^{\prime *}\right)=(n+m) \tilde{F}_{n, m},
$$

and, on the other hand,

$$
N \widetilde{F}_{n, m}=(\sigma \delta+\delta \sigma) \widetilde{F}_{n, m}=\delta\left(\sigma \widetilde{F}_{n, m}\right)=\delta H_{n, m} .
$$

So, we conclude that

$$
\tilde{F}_{n, m}=\delta\left(\frac{1}{(n+m)} H_{n, m}\right) \Rightarrow F=\delta H,
$$

that is, any $\delta$-closed function with ghost number different from zero is locally $\delta$ exact. This statement by no means signifies that this can occur globally. Indeed, due to the fact that the contracting homotopy $\sigma$ is only defined locally, there may appear global obstructions which do not allow us to patch together the results obtained in the local charts. As a consequence, a global expression for $H$ will not exist in general. This is precisely what happens in the ordinary de Rham cohomology, for which the cohomology spaces are intimately related with the topology of the manifold. In this sense, the operators $\delta, \sigma$, and $N$ can be viewed as some sort of exterior derivative $d$, its adjoint $d^{*}$ and the corresponding Laplacian $d d^{*}+d^{*} d$.

This result can be equivalently expressed by means of the following decomposition theorem, analogous to the Hodge decomposition in the ordinary de Rham cohomology. Given a function $F\left(C_{s}, C_{s}^{*}\right)$, it can be locally written as

$$
F=F_{0}+\delta A+\sigma B
$$

with $F_{0} \in$ ker $N$. To see this, consider the expansion (2.12) and apply the operator $N$ to it. Taking into account the relation (2.13) we have, for every $\widetilde{F}_{n, m}$

$$
\tilde{F}_{n, m}=N\left(\frac{1}{(n+m)} \tilde{F}_{n, m}\right)=\sigma\left[\delta \frac{1}{(n+m)} \tilde{F}_{n, m}\right]+\delta\left[\sigma \frac{1}{(n+m)} \tilde{F}_{n, m}\right]=\sigma B_{n, m}+\delta A_{n, m},
$$

and, therefore, $F=F_{0}+\delta A+\sigma B$, as we have said.

Now, if $\delta F=0$, gh $F \neq 0$, we have

$$
F=\delta A+\sigma B \Rightarrow \delta F=\delta(\delta A+\sigma B)=\delta \sigma B=0 .
$$

But, then

$$
N B=(\delta \sigma+\sigma \delta) B=\sigma \delta B \Rightarrow B=\sigma \widetilde{B} \Rightarrow \sigma B=0,
$$

and, as a result, $F=\delta A$, locally. 
Therefore, we conclude that the cohomology spaces $H_{k}(\delta)$ for $k \neq 0$ will be trivial if $\sigma$ can be defined globally, that is, if the local expressions of $H$ in Eq. (2.14) can be patched together without trouble.

\section{PERTURBATIVE SOLUTION OF THE MASTER EQUATION}

In this section we describe an alternative proof of the existence theorem ${ }^{11-13}$ of a solution of the master equation for a generic gauge theory in the framework of perturbation theory.

Let us consider a generic $L$ th stage reducible gauge theory with a classical action $S_{\mathrm{cl}}$, given by a power series expansion in some coupling constant $g$ of the form

$$
S_{\mathrm{cl}}=S_{\mathrm{cl}}^{(0)}\left[\phi^{2}\right]+S_{\mathrm{cl}}\left[g, \phi^{3}\right]+S_{\mathrm{cl}}\left[g^{2}, \phi^{4}\right]+\cdots,
$$

for which the gauge transformations can be written as

$$
\delta \phi^{i}=\widetilde{R}_{0 \alpha_{0}}^{i}[\phi] \epsilon^{\alpha_{0}}
$$

The form of the classical action (3.1), together with the boundary condition (A1) imposed by the classical limit, suggest that the proper solution of the master equation $S\left[\Phi, \Phi^{*}\right]$ should have also a similar power series expansion

$$
S\left[\Phi, \Phi^{*}\right]=\sum_{n=0} S_{n}\left[g^{n} ; \varphi\right], \quad \varphi \equiv\left\{\Phi, \Phi^{*}\right\} .
$$

Moreover, as we shall show in Appendix B, a generic term $S_{n}$ in this expansion could be taken to be homogeneous of degree $n+2$ in all the fields, i.e., of the form $S_{n}\left[g^{n} ; \varphi^{n+2}\right]$. This particular form implies in turn that we can assume a power series expansion for a generic structure function $\widetilde{F}_{i_{1} \cdots i_{n}}[\phi]$ with $n$ index of the form

$$
\widetilde{F}_{i_{1} \cdots i_{n}}[\phi]=\sum_{m=0} F_{i_{1} \cdots i_{n}}\left[g^{n-2+m}, \phi^{m}\right], \quad n \geqslant 2
$$

Although this statement is not fundamental in this section, it will be crucial in the proof of the locality of the proper solution.

Now, taking into account of the perturbative expansion of the proper solution (3.3), the conditions on $S_{n}$ imposed by the master equation, as follows by simple power counting arguments, are

$$
(S, S)=0 \Leftrightarrow \sum_{k} \sum_{l}\left(S_{k}, S_{l}\right)=\sum_{n=0}\left(\sum_{k=0}^{n}\left(S_{k}, S_{n-k}\right)\right)=0,
$$

which imply the recursive relations

$$
\left(S_{0}, S_{n}\right)+\frac{1}{2} \sum_{k=1}^{n-1}\left(S_{k}, S_{n-k}\right)=0, \quad \forall n=0,1, \ldots
$$

For $n=0, \mathrm{Eq} .(3.5)$ reads

$$
\left(S_{0}, S_{0}\right)=0
$$

Thus, at the lowest order in $g$, we must look for a "free" proper solution of the master equation, verifying the so-called classical limit. Using the results derived in Appendix B we can write 


$$
S_{0}\left[\Phi, \Phi^{*}\right]=S_{\mathrm{cl}}^{(0)}\left[\phi^{2}\right]+\sum_{s=0}^{L} C_{s-1, \alpha_{s-1}}^{*} R_{s \alpha_{s}}^{\alpha_{s-1}}\left[g^{0}, \phi^{0}\right] C_{s}^{\alpha_{s}},
$$

where $R_{s \alpha_{s}}^{\alpha_{s-1}}\left[g^{0}, \phi^{0}\right]$ stand for the free part of the generators of the gauge algebra. Note that this free part of the generators, in the framework of perturbation theory, should have the same rank as that of the generators of the complete theory $\widetilde{R}_{s \alpha_{s}}^{\alpha_{s-1}}[\phi]$ in order the spectrum of the free and the interacting theory coincide, i.e.,

$$
\operatorname{rank} \widetilde{R}_{s \alpha_{s}}^{\alpha_{s-1}}[\phi]=\operatorname{rank} R_{s \alpha_{s}}^{\alpha_{s-1}}\left[g^{0}, \phi^{0}\right], \quad s=0, \ldots, L .
$$

This condition guarantees that the free solution of the master equation (3.7) is proper. Therefore, from now on we will always assume it.

In order to show the existence of $S_{n}$ for a generic $n$, it will be useful to introduce the BRST transformation associated with the free part of the proper solution $S_{0}$

$$
\delta F=(-1)^{F}\left(F, S_{0}\right) .
$$

This transformation is nilpotent, as a consequence of Eq. (3.6) and the Jacobi identity for the antibracket, and increases the ghost number by one unit. With this definition the recursive relations $(3.5)$ can be written as

$$
\delta S_{n}+Q_{n}\left(S_{n-1}, \ldots, S_{1}\right)=0,
$$

where the quantities $Q_{n}$ are defined by

$$
Q_{n}\left(S_{n-1}, \ldots, S_{1}\right)=\frac{1}{2} \sum_{k=1}^{n-1}\left(S_{k}, S_{n-k}\right), \quad n \geqslant 2, \quad \text { gh } Q_{n}=1
$$

For $n=1$, the corresponding equation reflects the invariance of the term $S_{1}$ under the free BRST transformation (3.8). The explicit form for $S_{1}$, worked out in Ref. 4, reads (We refer the reader to this reference for further explanation of our conventions and notations.)

$$
\begin{aligned}
S_{1}= & S_{\mathrm{cl}}\left[g, \phi^{3}\right]+\sum_{s=0}^{L} C_{s-1, \alpha_{s-1}}^{*} R_{s \alpha_{s}}^{\alpha_{s-1}[g, \phi] C_{s}^{\alpha_{s}}} \\
& +\frac{1}{2} \sum_{s=0}^{L} \sum_{t=0}^{L}(-1)^{s(t+1)} C_{s+t, \alpha_{s+t}}^{*} U_{s t+\alpha_{t} \alpha_{s}}^{\alpha_{s+t}}\left[g, \phi^{0}\right] C_{s}^{\alpha_{s}} C_{t}^{\alpha_{t}} \\
& +\frac{1}{2} \sum_{s=0}^{L} \sum_{t=0}^{L}(-1)^{s(t+1)} C_{s-1, \alpha_{s-1}}^{*} C_{t-1, \alpha_{t-1}}^{*} V_{t s, \alpha_{s+t+1} \alpha_{t-1} \alpha_{s-1}}^{L}\left[g, \phi^{0}\right] C_{s+t+1}^{\alpha_{s+t+1}} .
\end{aligned}
$$

For $n \geqslant 2$, it is not obvious that the equations (3.9) could be solved. The necessary and sufficient conditions ensuring the existence of a perturbative solution of the master equation are that $Q_{n}$ be $\delta$ closed and the cohomology group of $\delta$ at ghost number one, $H_{1}(\delta)$, be zero

$$
H_{1}(\delta)=0 \text {. }
$$

Let us first prove $H_{1}(\delta)=0$ using the general results obtained in Sec. II. To this end, consider the contracting homotopy $\sigma$ defined there. As we have said, if the operator $\sigma$ can be defined globally, that is, if the local expressions of $H$ in Eq. (2.14) can be patched together without trouble, the cohomology spaces $H_{k}(\delta)$ for $k \neq 0$ will be trivial. This is what happens for 
the $L$ th stage reducible free gauge theory we are dealing with. Indeed, consider the free part of the generators of the gauge algebra $R_{s \alpha_{s}}^{\alpha_{s-1}}\left[g^{0}, \phi^{0}\right], s=0, \ldots, L$, separate the independent components $R_{A_{s-1}}^{\alpha_{s-1}}\left[g^{0}, \phi^{0}\right]$ and at each level complete these sets with vectors $R_{a_{s-1}}^{\alpha_{s-1}}\left[g^{0}, \phi^{0}\right]$ in such a way that the matrices

$$
R_{s B_{s-1}}^{\alpha_{s-1}} \equiv\left(R_{B_{s-1}}^{\alpha_{s-1}}, R_{b_{s-1}}^{\alpha_{s-1}}\right)=\left(\begin{array}{ll}
R_{B_{s-1}}^{A_{s-1}} & R_{B_{s-1}}^{a_{s-1}} \\
R_{b_{s-1}}^{A_{s-1}} & R_{b_{s-1}}^{a_{s-1}}
\end{array}\right)
$$

be invertible, i.e., there exist matrices $Q_{s \beta_{s-1}}^{\alpha_{s-1}}$ such that $Q_{s \gamma_{s-1}}^{\alpha_{s-1}} R_{s \beta_{s-1}}^{\gamma_{s-1}}=\delta_{\beta_{s-1}}^{\alpha_{s-1}}$.

Next, consider the canonical transformation, in the antibracket sense

$$
\begin{aligned}
& C_{s}^{\prime \alpha_{s}}=Q_{s \beta_{s}}^{\alpha_{s}} C_{s}^{\beta_{s}}=\left(Q_{\beta_{s}}^{A_{s}}, Q_{\beta_{s}}^{a_{s}}\right) C_{s}^{\beta_{s}} \equiv\left(C_{s}^{A_{s}}, C_{s}^{a_{s}}\right), \quad s=-1,0, \ldots, L, \\
& C_{s \alpha_{s}}^{\prime *}=C_{s \beta_{s}}^{*} R_{s \alpha_{s}}^{\beta_{s}}=C_{s \beta_{s}}^{*}\left(R_{A_{s}}^{\beta_{s}}, R_{a_{s}}^{\beta_{s}}\right) \equiv\left(C_{s A_{s}}^{*}, C_{s a_{s}}^{*}\right), \quad s=-1,0, \ldots, L .
\end{aligned}
$$

In terms of these new fields the free proper solution (3.7) turns out to be

$$
S_{0}=S_{\mathrm{cl}}^{(0)}\left(\phi^{A}-1\right)+\sum_{s=0}^{L} C_{s-1, a_{s-1}}^{*} \delta_{A_{s}}^{a_{s-1}} C_{s}^{A_{s}},
$$

which is precisely of the form (2.9) presented in Sec. II. What is important to note here is that, due to the fact that the matrices $Q_{s \beta_{s-1}}^{\alpha_{s-1}}, R_{s \beta_{s-1}}^{\alpha_{s-1}}$ do not depend on the fields, the canonical transformation (3.13) and the expression (3.14) for $S_{0}$ are valid everywhere and cover all the space of fields and antifields. On the other hand, the coordinate change (2.10) from the fields $\phi^{A-1}$ to the free equations of motion is also globally invertible. This is so because the Jacobian of this change (2.11) is the operator defining the free part of the classical action in the space of the gauge invariant fields $\phi^{A}-1$, which is independent of the fields and invertible. Consequently, the operators $\sigma$ and $N$ introduced above have well defined global expressions and, as a result, the local $\delta$-exact pieces $F=\delta H$ in (2.14) can be patched together to form a globally defined object.

Therefore, we conclude that the antibracket BRST cohomology defined in the space of all functionals (local and nonlocal ones) of the free proper solution $S_{0}$ under consideration is trivial except at ghost number 0 , i.e.,

$$
H_{k}(\delta)=0, \quad \forall k \neq 0,
$$

and that, in particular, the condition (3.12), $H_{1}(\delta)=0$, is verified.

To complete the proof, let us check by induction that $Q_{n}$ are $\delta$ closed for all $n$. Consider the first nonvanishing $Q_{n}$, i.e., $Q_{2}$. Its BRST variation is

$$
\delta Q_{2}=\left(S_{1}, \delta S_{1}\right)=0,
$$

which is zero by virtue of the BRST invariance of $S_{1}$. Taking into account that (3.12) is verified, this allows us to solve the equation for $S_{2}$ as

$$
\delta S_{2}+Q_{2}=\delta S_{2}-\delta F_{2}=0 \Rightarrow S_{2}=F_{2}+\delta \widetilde{F}_{2} .
$$

Now, consider the BRST transformation of the term $Q_{n}$. From Eqs. (3.8) and (3.10), we obtain 


$$
\delta Q_{n}=\frac{1}{2} \sum_{k=1}^{n-1}\left[\left(\delta S_{k}, S_{n-k}\right)-\left(S_{k}, \delta S_{n-k}\right)\right]
$$

If we assume that the master equation has been solved up to order $n-1$ (which is valid for $n-1=2$ ), we have

$$
\delta Q_{n}=\frac{1}{2} \sum_{k=1}^{n-1}\left[\left(S_{n-k}, Q_{k}\right)+\left(S_{k}, Q_{n-k}\right)\right]=\sum_{k=1}^{n-1}\left(S_{k}, Q_{n-k}\right) .
$$

Finally, taking into account of the definition of $Q_{k}, Q_{n-k}$ and making further index redefinitions we arrive at

$$
\delta Q_{n}=\frac{1}{3} \sum_{k=1}^{n-1} \sum_{l=1}^{n-k-l}\left[\left(S_{n-k-l},\left(S_{k}, S_{l}\right)\right)+(\text { cicl. perm. })\right]=0,
$$

which is zero by virtue of the Jacobi identity for the antibracket.

In summary, we have shown that the consistency conditions $\delta Q_{n}=0$ and (3.12) are verified. This fact implies that the recursive relations (3.9) can be solved order by order in perturbation theory and ensures the existence of a perturbative solution of the master equation.

\section{LOCALITY OF THE PROPER SOLUTION}

In Sec. III we have seen that it is possible to construct a perturbative solution of the master equation. Moreover, the arguments presented in Appendix A show that this solution can be taken to be proper and verify the classical limit. The question of the locality, however, has remained open until now.

In this section we will see that, if the following conditions are verified, namely,

(a) the classical action $S_{\mathrm{cl}}$ is local,

(b) the generators of the gauge algebra $\widetilde{R}_{s \alpha_{s}}^{\alpha_{s-1}}, s=0, \ldots, L$, are local, and

(c) the free part of the generators of the gauge algebra $R_{s \alpha_{s}}^{\alpha_{s-1}}\left[g^{0}, \phi^{0}\right], s=0, \ldots, L$, are locally complete,

the structure functions and, consequently, the full proper solution $S$, can be taken to be local. These conditions turn out to be equivalent as those presented in Ref. 14.

The condition of local completeness of the free part of the generators of the gauge algebra requires further explanation. It is said that the free part of the generators of the gauge transformations, $R_{0 \alpha_{0}}^{i}\left[g^{0}, \phi^{0}\right]$, are locally complete if any local function $\lambda^{i}$ verifying

$$
\lambda^{i} S_{\mathrm{cl}, i}^{(0)}=0
$$

can be expressed as

$$
\lambda^{i}=R_{0 \alpha_{0}}^{i}\left[g^{0}, \phi^{0}\right] \lambda^{\alpha_{0}}+T^{i j} S_{\mathrm{cl}, j}^{(0)},
$$

with $\lambda^{\alpha_{0}}, T^{i j}$ local functions and $T^{i j}=-T^{j i}$. In much the same way, the free part of the reducibility functionals, $R_{s \alpha_{s}}^{\alpha_{s-1}}\left[g^{0}, \phi^{0}\right], s=1, \ldots, L$, are said to be locally complete if any local function $\lambda^{\alpha_{s}}$ which verifies

$$
R_{s \alpha_{s}}^{\alpha_{s-1}}\left[g^{0}, \phi^{0}\right] \lambda^{\alpha_{s}}=M_{s}^{\alpha_{s-1} i} S_{\mathrm{cl}, i}^{(0)}
$$

can be written as 


$$
\lambda^{\alpha_{s}}=R_{s+1, \alpha_{s+1}}^{\alpha_{s}}\left[g^{0}, \phi^{0}\right] \lambda^{\alpha_{s+1}}+T^{\alpha_{s} i} S_{\mathrm{cl}, i}^{(0)},
$$

where the coefficients $\lambda^{\alpha_{s+1}}, T^{\alpha_{s} i}$, are also local functions.

To study the locality let us translate, first of all, the recurrent equations (3.9) to the structure functions. Assume a power series expansion for $S$ of the type (3.3), whose validity is studied in Appendix B. A homogeneous term $S_{n}$ of this expansion can be written in these conditions as

$$
S_{n}\left[g^{n}, \varphi^{n+2}\right]=\sum_{k=0}^{n+2} F_{i_{1} \cdots i_{k}}\left[g^{n}, \phi^{n+2-k}\right] \bar{\varphi}^{i_{1}} \cdots \bar{\varphi}^{i_{k}},
$$

where the fields $\bar{\varphi}^{i}$ stand for all the fields and antifields of the minimal sector except the classical fields $\phi$. The coefficients $F_{i_{1} \cdots i_{k}}\left[g^{n}, \phi^{n+2-k}\right]$, the $n$th terms in the developments of the structure functions in the coupling constant (3.4), are polynomials in the classical fields and their derivatives. Therefore, we can write $S_{n}$ in a more compact form as

$$
S_{n}=\sum F_{i_{1} \cdots i_{n+2}}\left[g^{n}\right] \varphi^{i_{1}} \cdots \varphi^{i_{n+2}}
$$

or equivalently

$$
S_{n}=\sum \frac{1}{j ! k !} C_{t_{1} \beta_{t_{1}}}^{*} \cdots C_{t_{j} \beta_{t_{j}}}^{*} F_{\alpha_{s_{1}} \cdots \alpha_{s_{k}}}^{\beta_{t_{1}} \cdots \beta_{t_{j}}}\left[g^{n}\right] C_{s_{1}}^{\alpha_{s_{1}}} \cdots C_{s_{k}}^{\alpha_{s_{k}}}
$$

with the restrictions $j+k=n+2$, gh $S_{n}=0$. The coefficients $F_{\alpha_{s_{1}}}^{\beta_{t_{1}} \cdots}\left[g^{n}\right]$, which are differential operators in general, do not depend on the classical fields and their indices have the corresponding generalized symmetry. Here, as always, $\left(C_{s}, C_{s}^{*}\right), s=-1,0, \ldots, L$, stand for all the fields and antifields of the minimal sector.

To determine the form of the recurrent equations for the structure functions from the recursive relations (3.9), we need the action of $\delta$ on all the fields. From its definition (3.8) and the form of $S_{0}(3.7)$, we have, in compact form

$$
\begin{gathered}
\delta C_{s}^{\alpha_{s}}=(-1)^{s+1} R_{s+1, \alpha_{s+1}}^{\alpha_{s}}\left[g^{0}, \phi^{0}\right] C_{s+1}^{\alpha_{s+1}}, \quad s=-1, \ldots, L, \\
\delta C_{s \alpha_{s}}^{*}=C_{s-1, \alpha_{s-1}}^{*} R_{s \alpha_{s}}^{\alpha_{s-1}}\left[g^{0}, \phi^{0}\right], \quad s=-1, \ldots, L,
\end{gathered}
$$

where we have made the identification

$$
\delta C_{-1 \alpha_{-1}}^{*}=\delta \phi_{i}^{*}=C_{-2, \alpha_{-2}}^{*} R_{-1 \alpha_{-1}}^{\alpha_{-2}}\left[g^{0}, \phi^{0}\right] \equiv S_{\mathrm{cl}, i}^{(0)} .
$$

The actuation of $\delta$ on $S_{n}$ reads now

$$
\begin{aligned}
\delta S_{n}= & \sum \frac{1}{(j-1) ! k !} C_{t_{1} \beta_{t_{1}}}^{*} \cdots C_{t_{j} \beta_{t_{j}}}^{*} R_{t_{1}+1, \beta_{t_{1}+1}}^{\beta_{t_{1}}} F_{\alpha_{s_{1}} \cdots \alpha_{s_{k}}}^{\beta_{t_{1}+1} \cdots \beta_{t_{j}}}\left[g^{n}\right] C_{s_{1}}^{\alpha_{s_{1}}} \cdots C_{s_{k}}^{\alpha_{s_{k}}} \\
& +\sum \frac{1}{j !(k-1) !} C_{t_{1} \beta_{t_{1}}}^{*} \cdots C_{t_{j} \beta_{t_{j}}}^{*} F_{\alpha_{s_{1}} \cdots \alpha_{s_{k}-1}}^{\beta_{t_{1}} \cdots \beta_{t_{j}}}\left[g^{n}\right] R_{s_{k} \alpha_{s_{k}}}^{\alpha_{s_{k}-1}} C_{s_{1}}^{\alpha_{s_{1}} \cdots C_{s_{k}}^{\alpha_{s_{k}}}}
\end{aligned}
$$

The inhomogeneous term $Q_{n}$ will have a similar expansion as that of $S_{n}$ (4.1) 


$$
Q_{n}=\sum C_{t_{1} \beta_{t_{1}}}^{*} \cdots C_{t_{j} \beta_{t_{j}}}^{*} Q_{\alpha_{s_{1}} \cdots \alpha_{s_{k}}}^{\beta_{t_{1}} \cdots \beta_{t_{j}}}\left[g^{n}\right] C_{s_{1}}^{\alpha_{s_{1}} \cdots C_{s_{k}}^{\alpha_{s_{k}}}}
$$

with the restrictions $j+k=n+2$, gh $Q_{n}=1$. Finally, collecting all these expressions we arrive at the following recurrent equations for the structure functions

$$
\frac{1}{(j-1) ! k !} R_{t_{1}+1, \beta_{t_{1}+1}}^{\left[\beta_{t_{1}}\right.} \quad F_{\alpha_{s_{1}} \cdots \alpha_{s_{k}}}^{\left.\beta_{t_{1}+1} \cdots \beta_{t_{j}}\right]}\left[g^{n}\right]=\frac{(-1)}{j !(k-1) !} F_{\left[\alpha_{s_{1}} \cdots \alpha_{s_{k}-1}\right.}^{\beta_{t_{1}} \cdots \beta_{t_{j}}}\left[g^{n}\right] R_{\left.s_{k} \alpha_{s_{k}}\right]}^{\alpha_{s_{k}-1}}+Q_{\alpha_{s_{1}} \cdots \alpha_{s_{k}}}^{\beta_{t_{1}} \cdots \beta_{t_{j}}}\left[g^{n}\right],
$$

where the square brackets mean suitable symetrization of the enclosed free indices.

Let us analyze these equations and the form of the terms $S_{n}$ in more detail. As we argue in Appendix A, the lowest order terms in the antifields of a solution of the master equation verifying the boundary conditions (A3) and (A4) read, Eq. (A5),

$$
S_{n}\left[\Phi, \Phi^{*}\right]=S_{\mathrm{cl}}\left[g^{n}, \phi^{n+2}\right]+\sum_{s=0}^{L} C_{s-1, \alpha_{s-1}}^{*} R_{s \alpha_{s}}^{\alpha_{s-1}}\left[g^{n}, \phi^{n}\right] C_{s}^{\alpha_{s}}+O\left[\left(C^{*}\right)^{2}\right]
$$

Therefore, to guarantee the locality of $S_{n}$ we must impose, at least, that $S_{\mathrm{cl}}$ and the generators of the gauge algebra to be local, hence, conditions (a) and (b).

These conditions ensure automatically that $S_{0}$ is local and, as a consequence, that the operator $\delta$ (3.8) transforms local expressions into local expressions. With respect to the higher order terms, we will consider first of all $S_{1}$. The recurrent equations for $S_{1}$ can be written as ${ }^{3,4}$

$$
\begin{aligned}
& R_{0 \alpha_{0}, j}^{i}[g, \phi] R_{0 \beta_{0}}^{j}\left[g^{0}, \phi^{0}\right]-R_{0 \beta_{0}, j}^{i}[g, \phi] R_{0 \alpha_{0}}^{j}\left[g^{0}, \phi^{0}\right]=R_{0 \gamma_{0}}^{i}\left[g^{0}, \phi^{0}\right] U_{00 \alpha_{0} \beta_{0}}^{\gamma_{0}}\left[g, \phi^{0}\right], \\
& R_{s \alpha_{s}, j}^{\alpha_{s-1}}[g, \phi] R_{0 \alpha_{0}}^{j}\left[g^{0}, \phi^{0}\right]+U_{0, s-1, \beta_{s-1}, \alpha_{0}}^{\alpha_{s-1}}\left[g, \phi^{0}\right] R_{s \alpha_{s}}^{\beta_{s-1}}\left[g^{0}, \phi^{0}\right]=R_{s \beta_{s}}^{\alpha_{s-1}}\left[g^{0}, \phi^{0}\right] U_{0 s \alpha_{s} \alpha_{0}}^{\beta_{s}}\left[g, \phi^{0}\right], \\
& U_{t s \alpha_{s} \alpha_{t}}^{\alpha_{s+l}}\left[g, \phi^{0}\right] R_{t+1, \alpha_{t+1}}^{\alpha_{t}}\left[g^{0}, \phi^{0}\right]+(-1)^{t+1} U_{t+1, s-1, \alpha_{s-1}, \alpha_{t+1}}^{\alpha_{s+l}}\left[g, \phi^{0}\right] R_{s \alpha_{s}}^{\alpha_{s-1}}\left[g^{0}, \phi^{0}\right] \\
& =R_{s+t+1, \alpha_{s+t+1}}^{\alpha_{s+t}}\left[g^{0}, \phi^{0}\right] U_{t+1, s \alpha_{s} \alpha_{t+1}}^{\alpha_{s+t}}\left[g, \phi^{0}\right], \\
& R_{s \alpha_{s}}^{\alpha_{s-1}}\left[g^{0}, \phi^{0}\right] R_{s+1, \alpha_{s+1}}^{\alpha_{s}}[g, \phi]+R_{s \alpha_{s}}^{\alpha_{s-1}}[g, \phi] R_{s+1, \alpha_{s+1}}^{\alpha_{s}}\left[g^{0}, \phi^{0}\right]=S_{\mathrm{cl}, i}^{(0)} V_{0 s \alpha_{s+1}^{i \alpha_{s-1}}}^{\alpha}\left[g, \phi^{0}\right], \\
& V_{t, s-1, \alpha_{s+t}}^{\alpha_{t-1} \alpha_{s-2}}\left[g, \phi^{0}\right] R_{s+t+1, \alpha_{s+t+1}}^{\alpha_{s+t}}\left[g^{0}, \phi^{0}\right]-(-1)^{t} R_{s-1, \alpha_{s-1}}^{\alpha_{s-2}}\left[g^{0}, \phi^{0}\right] V_{t s, \alpha_{s+t+1}}^{\alpha_{t-1} \alpha_{s-1}}\left[g, \phi^{0}\right] \\
& =R_{t \alpha_{t}}^{\alpha_{t-1}}\left[g^{0}, \phi^{0}\right] V_{t+1, s-1, \alpha_{s+t+1}}^{\alpha_{t} \alpha_{s-2}}\left[g, \phi^{0}\right]
\end{aligned}
$$

where $U_{s l}\left[g, \phi^{0}\right], V_{s t}\left[g, \phi^{0}\right]$ are the structure constants appearing in Eq. (3.11).

Consider now the equations (4.4) which determine the structure constants $U_{00}\left[g, \phi^{0}\right]$ from the knowledge of the lowest order terms of the generators $\widetilde{R}_{0 \alpha_{0}}^{i}$. By hypothesis (b), the left-hand side of these equations are local expressions verifying

$$
S_{\mathrm{cl}, i}^{(0)}\left(R_{0 \alpha_{0}, j}^{i}[g, \phi] R_{0 \beta_{0}}^{j}\left[g^{0}, \phi^{0}\right]-R_{0 \beta_{0}, j}^{i}[g, \phi] R_{0 \alpha_{0}}^{j}\left[g^{0}, \phi^{0}\right]\right)=0 .
$$

Moreover, by local completeness of $R_{0 \beta_{0}}^{i}\left[g^{0}, \phi^{0}\right]$, this expression can be expressed as a combination of them with local coefficients. Hence, the structure functions $U_{00}\left[g, \phi^{0}\right]$, which are nothing but these coefficients, can be taken to be local. 
The group of equations (4.5) determines $U_{0 s}\left[g, \phi^{0}\right]$ once $U_{0, s-1}\left[g, \phi^{0}\right]$ are known. Suppose, thus, that $U_{0, s-1}\left[g, \phi^{0}\right]$ are local (which is valid for $U_{00}\left[g, \phi^{0}\right]$ ). Then, the left-hand side of these relations is a local expression which verifies

$$
R_{s-1, \alpha_{s-1}}^{\alpha_{s-2}}\left[g^{0}, \phi^{0}\right]\left(R_{s \alpha_{s}, j}^{\alpha_{s-1}}[g, \phi] R_{0 \alpha_{0}}^{j}\left[g^{0}, \phi^{0}\right]+U_{0, s-1, \beta_{s-1}, \alpha_{0}}^{\alpha_{s-1}}\left[g, \phi^{0}\right] R_{s \alpha_{s}}^{\beta_{s-1}}\left[g^{0}, \phi^{0}\right]\right)=0 .
$$

Therefore, by local completeness, the quantity inside the bracket can be expressed as a combination of $R_{s \alpha_{s}}^{\alpha_{s-1}}\left[g^{0}, \phi^{0}\right]$ with local coefficients. As a consequence, the structure functions $U_{05}\left[g, \phi^{\circ}\right]$ can also be chosen to be local.

Finally, the equations (4.6) allow us to determine recursively the remaining $U_{t+1, s}\left[g, \phi^{0}\right]$ at lowest order in perturbation theory from the knowledge of the $U$-structure functions with lowest or equal index $U_{t+1, s-1}\left[g, \phi^{0}\right], U_{t s}\left[g, \phi^{0}\right]$. What is important to note here is that the unknown quantity $U_{t+1, s}\left[g, \phi^{0}\right]$ appears in the equation in the form $R_{s+t+1}\left[g^{0}, \phi^{0}\right] U_{t+1, s}\left[g, \phi^{0}\right]$, while the remaining expression is a local quantity, by recursive hypothesis, which contracted with $R_{s+i}\left[g^{0}, \phi^{0}\right]$ vanishes. So, applying the same arguments as above, we conclude that $U_{t+1, s}\left[g, \phi^{0}\right]$ can also be taken local. Proceeding in much the same way one can see from Eqs. (4.7) and (4.8) that $V_{s t}\left[g, \phi^{0}\right]$ at this order can be chosen local. Therefore, with such selections $S_{1}$ turns out to be local.

From the above analysis, it should be clear how to proceed in the general case. Suppose that, up to order $n-1$, all the terms $S_{k}, k=0, \ldots, n-1$, have been constructed local. Being all of them local, $Q_{n}$ and the corresponding coefficients $Q \cdots$ appearing in the expansion (4.2) are also local. On the other hand, assume that the coefficients

$$
F_{\alpha_{s_{1}} \cdots \alpha_{s_{k-1}}}^{\beta_{t_{1}} \cdots \beta_{t_{j}}}\left[g^{n}\right]
$$

have been determined as local terms. In such conditions, the right-hand side of Eq. (4.3) is a local expression which vanishes upon application of $R_{s \alpha_{s}}^{\alpha_{s-1}}\left[g^{0}, \phi^{0}\right]$. By hypothesis (c) this expression can be written as a combination of these generators with local coefficients. Therefore, the coefficients

$$
F_{\alpha_{s_{1}} \cdots \alpha_{s_{k}}}^{\beta_{t_{1}+1} \cdots \beta_{t_{j}}}\left[g^{n}\right]
$$

with index higher than or equal to (4.9), can be constructed locally. Applying this argument recursively, we conclude that all the coefficients appearing in the $n$th term $S_{n}$ of the proper solution of the master equation can be taken to be local. This completes the proof of the locality of the proper solution $S$ in the framework of perturbation theory.

\section{CONCLUSIONS}

In this article we have analyzed the antibracket BRST cohomology defined in the space of all functionals, especially the cohomology of the free part of the proper solution of the master equation, $S_{0}$, using homological techniques. The main result, namely, that the cohomological spaces $H_{k}(\delta)$ for $k \neq 0$ are trivial in the free case, has allowed us to find a perturbative solution of the master equation, as well as to obtain the form of the power series expansions in the coupling constant for the structure functions, i.e.,

$$
\tilde{F}_{i_{1} \cdots i_{n}}[\phi]=\sum_{m=0} F_{i_{1} \cdots i_{n}}\left[g^{n-2+m}, \phi^{m}\right], \quad n>2 .
$$

The question of the locality of the solution obtained has also been considered. Our study has shown that provided (i) the classical action is local, (ii) the generators of the gauge algebra 
$\widetilde{K}_{s \alpha_{s}}^{\alpha_{s-1}}, s=0, \ldots, L$, are local, and (iii) the free part of these generators is locally complete, it is possible to choose the structure functions defining the gauge algebra and, as a result, the proper solution of the master equation, to be local.

The problem of a perturbative solution of the quantum master equation and its locality is a subject presently under study.

\section{ACKNOWLEDGMENTS}

One of us (J. G.) ackowledges Professor K. Nakanishi for discussions and critical reading of the manuscript and Professor R. Sakaki for comments. This work has been partially supported by CICYT Project No. AEN89-0347. J. P. acknowledges a fellowship from Ministerio de Educación y Ciencia.

\section{APPENDIX A: PROPERNESS AND CLASSICAL LIMIT OF S IN PERTURBATION THEORY}

In this Appendix, we analyze the questions of the properness and the classical limit of the solution of the master equation in the framework of perturbation theory. As we know from the work of Batalin and Vilkovisky, ${ }^{2}$ the content of the minimal set of fields $\Phi^{A}$ and the boundary conditions to the master equation are determined from two requeriments: the correctness of the classical limit and the nondegeneracy of the functional integral.

The correctness of the classical limit translates to the following boundary condition for $S$

$$
\left.S\left[\Phi, \Phi^{*}\right]\right|_{\Phi *=0}=S_{\mathrm{cl}}\left(\phi^{i}\right)
$$

The nondegeneracy of the functional integral imposes that $S$ be the proper solution of the master equation. A solution is called proper if the rank of the Hessian at the stationary point of $S$ is the maximal possible (and equal to the number of fields $\Phi^{A}$ ). Furthermore, it is possible to show that this requirement turns out to be equivalent to the boundary conditions

$$
\left.\frac{\partial_{l} \partial_{r} S}{\partial C_{s-1, \alpha_{s-1}}^{*} \partial C_{s}^{\alpha_{s}}}\right|_{\Phi *=0}=\widetilde{R}_{s \alpha_{s}}^{\alpha_{s-1}}[\phi], \quad s=0, \ldots, L,
$$

$\widetilde{R}_{s \alpha_{s}}^{\alpha_{s-1}}[\phi]$ being the generators of the gauge algebra. In addition, the requirement of nondegeneracy imposes also some restrictions on the gauge fermion, which in the framework of perturbation theory were considered in Ref. 4 and that will not be analyzed here.

Now, let us translate these conditions to our particular case. The boundary condition imposed by the correctness of the classical limit can be expressed perturbatively as

$$
\left.S_{n}\left[\Phi, \Phi^{*}\right]\right|_{\Phi *=0}=S_{\mathrm{cl}}\left[g^{n}, \phi^{n+2}\right] .
$$

With respect to the nondegeneracy, taking into account of the form (B2) and (B6) of the generators of the gauge algebra given in Appendix B, the conditions (A2) read

$$
\left.\frac{\partial_{l} \partial_{r} S_{n}}{\partial C_{s-1, \alpha_{s-1}}^{*} \partial C_{s}^{\alpha_{s}}}\right|_{\Phi *=0}=R_{s \alpha_{s}}^{\alpha_{s-1}}\left[g^{n}, \phi^{n}\right], \quad s=0, \ldots, L .
$$

Finally, as a byproduct of this analysis, we can derive the form of the lowest order terms in the antifields of a generic term $S_{n}$ in the perturbative expansion of $S$. Indeed, from the boundary conditions (A3) and (A4) it is evident that 


$$
S_{n}\left[\Phi, \Phi^{*}\right]=S_{\mathrm{cl}}\left[g^{n}, \phi^{n+2}\right]+\sum_{s=0}^{L} C_{s-1, \alpha_{s-1}}^{*} R_{s \alpha_{s}}^{\alpha_{s-1}}\left[g^{n}, \phi^{n}\right] C_{s}^{\alpha_{s}}+O\left[\left(C^{*}\right)^{2}\right],
$$

which is one of the expressions we use in Sec. IV to study the locality of the proper solution of the master equation in perturbation theory.

It is important to note that the requirement on the solution of the master equation to be proper is automatically verified if $S_{0}$ is constructed to be proper, at lcast in the framcwork of perturbation theory. Indeed, as we have said, the requirement of properness imposes the rank of the Hessian to be the maximal possible at the stationary point. Thus, suppose that $S_{0}$ is proper and consider the Hessian of the complete solution $S$. We have

$$
\left.\operatorname{Hess}(S)\right|_{\varphi}=\left.\operatorname{Hess}\left(S_{0}\right)\right|_{\varphi_{0}}+O(g)
$$

where $\varphi$ and $\varphi_{0}$ are the stationary points of $S$ and $S_{0}$, respectively, which are related by $\varphi=\varphi_{0}+O(g)$ in perturbation theory.

Since $S_{0}$ is proper, Hess $\left(S_{0}\right)$ has the maximum rank possible at the stationary point of $S_{0}$. The higher order terms in $g$ will have a rank which is smaller or equal than $\operatorname{rank}\left(\operatorname{Hess}\left(S_{0}\right)\right)$, but being at least of order $g$ they will not be able to reduce the rank of Hess $\left(S_{0}\right)$. Therefore,

$$
\operatorname{rank} \operatorname{Hess}(S)=\operatorname{rank} \operatorname{Hess}\left(S_{0}\right)=\text { maximal, }
$$

and, as a consequence, $S$ constructed through $S_{0}$ will be proper if $S_{0}$ is proper. In summary, the requirement of properness of $S_{0}$ determines the correct spectrum of fields and ensures the properness of the complete solution $S$ constructed through Eq. (3.9).

\section{APPENDIX B:}

Throughout this paper we have been assuming suitable power series expansions in the coupling constant for the structure functions, which have turned out to be very important in the derivation of the results. In this appendix we will justify this particular form.

Consider now the classical action (3.1) we are dealing with and the generators of their gauge transformations (3.2), $\widetilde{R}_{0 \alpha_{0}}^{i}$. The invariance of $S_{\mathrm{cl}}$ under these gauge transformations is expressed through the Noether identities

$$
\frac{\partial S_{\mathrm{cl}}}{\partial \phi^{i}} \widetilde{R}_{0 \alpha_{0}}^{i}=0
$$

Due to the form of the classical action it is obvious, by simple power counting in the coupling constant, that Eq. (B1) can only be verified if $\widetilde{R}_{0 \alpha_{0}}^{i}$ admits itself a power series expansion in it. Furthermore, by power counting in the classical fields, it is possible to show that a generic $n$th term of this expansion can be chosen to be homegeneous of degree $n$ in the classical fields. Therefore, the power series expansion for the generators of the gauge transformations can be taken of the form

$$
\tilde{R}_{0 \alpha_{0}}^{i}[\phi]=\sum_{n=0} R_{0 \alpha_{0}}^{i}\left[g^{n}, \phi^{n}\right] .
$$

In much the same way, by analyzing the $L$ th stage reducible gauge free theory given by $S_{\mathrm{cl}}^{(0)}$ one can see that, at zeroth order in the coupling constant, the reducibility functionals can be chosen to be independent of the classical fields $\phi$, i.e.,

$$
\left.\widetilde{R}_{s \alpha_{s}}^{\alpha_{s-1}}[\phi]\right|_{g=0}=R_{s \alpha_{s}}^{\alpha_{s-1}}\left[g^{0}, \phi^{0}\right] .
$$


The form of the generators (B2) and the boundary conditions (A3) and (A4) lead us to the following form for the $n$th term of the proper solution of the master equation

$$
S_{n}\left[g^{n}, \varphi\right]=S_{\mathrm{cl}}\left[g^{n}, \phi^{n+2}\right]+\phi_{i}^{*} R_{0 \alpha_{0}}^{i}\left[g^{n}, \phi^{n}\right] C_{0}^{\alpha_{0}}+\cdots .
$$

These lowest order terms appearing in $S_{n}$ are homogeneous of degree $n+2$ in all the fields, i.e., of the form $A\left[g^{n}, \varphi^{n+2}\right]$. Therefore, one would expect the remaining terms, and the complete $S_{n}$, to be of the same form, that is

$$
S_{n}=S_{n}\left[g^{n}, \varphi^{n+2}\right]
$$

Now, let us see that the form of the equations (3.9) enable us to choose $S_{n}$ in this particular form, which leads to the form (3.4) for the structure functions, as we shall show.

Indeed, consider, first of all, the lowest order term of the proper solution, $S_{0}$. Taking into account of the above results, in particular Eq. (B3), and the boundary conditions (A3) and (A4) derived in Appendix A, one concludes that the suitable form for the free proper solution $S_{0}$ is

$$
S_{0}\left[\Phi, \Phi^{*}\right]=S_{\mathrm{cl}}^{(0)}\left[\phi^{2}\right]+\sum_{s=0}^{L} C_{s-1, \alpha_{s-1}}^{*} R_{s \alpha_{s}}^{\alpha_{s-1}}\left[g^{0}, \phi^{0}\right] C_{s}^{\alpha_{s}},
$$

which is the expression (3.7) used in Sec. III and given also in Ref. 4. In particular, $S_{0}$ is homogeneous of degree 2 in all the fields, as expected. Due to this fact, the action of the operator $\delta$, Eq. (3.8), associated with $S_{0}$ does not increase the content of fields and the degree of the coupling constant, i.e.,

$$
\delta A\left[g^{n}, \varphi^{m}\right]=B\left[g^{n}, \varphi^{m}\right]
$$

Now, consider the first order term of the proper solution $S_{1}$, verifying $\delta S_{1}=0$. From the form (3.11) of this term as well as the relations (4.4)-(4.8) derived from $\delta S_{1}=0$ onc can sce that, indeed, $S_{1}$ can be taken to be homogeneous of degree 3 , in accordance with the general form (B4).

Once $S_{0}$ and $S_{1}$ are given, the terms $S_{n}, n \geqslant 2$, are determined from Eq. (3.9). For $n=2$, the corresponding equation is

$$
\delta S_{2}+1 / 2\left(S_{1}, S_{1}\right)=0 \Rightarrow \delta S_{2}+Q_{2}=0,
$$

where the content in fields and antifields of $Q_{2}$ can be derived as

$$
Q_{2}=1 / 2\left(S_{1}, S_{1}\right)=\frac{\partial_{r} S_{1}}{\partial \Phi^{A}} \frac{\partial_{l} S_{1}}{\partial \Phi_{A}^{*}} \Rightarrow Q_{2}=Q_{2}\left[g^{2}, \varphi^{A}\right]
$$

On the other hand, due to the actuation of $\delta$, Eq. (B5), and the fact that $H_{1}(\delta)=0$, we have

$$
Q_{2}=-\delta F_{2} \Rightarrow F_{2}\left[g^{2}, \varphi^{4}\right]
$$

and, as a consequence

$$
S_{2}=F_{2}\left[g^{2}, \varphi^{4}\right]+\delta \widetilde{F}_{2} .
$$

If $\widetilde{F}_{2}$ is chosen to be of the form $\widetilde{F}_{2}\left[g^{2}, \varphi^{4}\right], S_{2}$ will be in accordance with the general form (B4). Therefore, the precise statement is that $S_{2}$ can be chosen to be of this particular form. 
To conclude this argument, suppose that, up to order $n-1$, all the terms $S_{k}, k=0, \ldots, n-1$, have been taken of the form (B4). In such conditions, let us see that $S_{n}$ can be chosen to be of the same form. The equation for $S_{n}$ is

$$
\delta S_{n}+Q_{n}=0 \Rightarrow \delta S_{n}+1 / 2 \sum_{k=1}^{n-1}\left(S_{k}, S_{n-k}\right)=0
$$

A generic term of the summation defining $Q_{n}$ is of the form

$$
\left(S_{k}, S_{n-k}\right)=\frac{\partial_{r} S_{k}}{\partial \Phi^{A}} \frac{\partial_{l} S_{n-k}}{\partial \Phi_{A}^{*}}-\frac{\partial_{r} S_{k}}{\partial \Phi_{A}^{*}} \frac{\partial_{l} S_{n-k}}{\partial \Phi^{A}}=A\left[g^{n}, \varphi^{n+2}\right],
$$

which implies that $Q_{n}=Q_{n}\left[g^{n}, \varphi^{n+2}\right]$. Applying the same argument as for the case $n=2$, we conclude that $S_{n}$ can be taken of the form (B4), as we expected.

Now, we are in conditions to justify the generic form of the structure functions (3.4). Given a generic term $S_{n}\left[g^{n}, \varphi^{n+2}\right]$ of the proper solution, it will have an expansion of the type

$$
S_{n}=\sum_{k=0}^{n+2} F_{i_{1} \cdots i_{k}}\left[g^{n}, \phi^{n+2-k}\right] \bar{\varphi}^{i_{1}} \cdots \bar{\varphi}^{i_{k}}
$$

where $\bar{\varphi}$ stand for all the fields except the classical ones. The coefficients $F_{i_{1} \cdots i_{k}}\left[g^{n}, \phi^{n+2-k}\right]$ can be considered as the $n$th terms of the power series expansion of the structure function $\widetilde{F}_{i_{1} \cdots i_{k}}[\phi]$ going with a generic group $\bar{\varphi}^{i_{1}} \cdots \bar{\varphi}^{i_{k}}$ in the complete proper solution of the master equation $S$.

Therefore, following this reasoning, it is clear that we can assume power series expansions for the structure functions with $n$ index of the form

$$
\widetilde{F}_{i_{1} \cdots i_{n}}[\phi]=\sum_{m=0} F_{i_{1} \cdots i_{n}}\left[g^{n-2+m}, \phi^{m}\right], \quad n \geqslant 2,
$$

which are nothing but the form (3.4) assumed throughout this paper. In particular, the reducibility functionals $\widetilde{R}_{s \alpha_{s}}^{\alpha_{s-1}}[\phi]$, which correspond to the case $n=2$, are of the form

$$
\widetilde{R}_{s \alpha_{s}}^{\alpha_{s-1}}[\phi]=\sum_{n=0} R_{s \alpha_{s}}^{\alpha_{s-1}}\left[g^{n}, \phi^{n}\right]
$$

allowing to express the properness requirement (A2) of Appendix A in perturbation theory as (A4).

${ }^{1}$ C. Becchi, A. Rouet, and R. Stora, Ann. Phys. 98, 287 (1976); I. V. Tyutin, Lebedev preprint FIAN n.39 (1975) (unpublished).

${ }^{2}$ I. A. Batalin and G. A. Vilkovisky, Phys. Lett. B 102, 27 (1981); Phys. Rev. D 28, 2567 (1983).

${ }^{3}$ S. A. Frolov and A. A. Slavnov, Nucl. Phys. B 347, 333 (1990). See also A. A. Slavnov, Nucl. Phys. (Proc. Suppl.)

B 15, 107 (1990).

4J. Gomis and J. Paris, Nucl. Phys. B 368, 311 (1992).

${ }^{3}$ T. Kugo and I. Ojima, Suppl. Prog. Theor. Phys. 66, 1 (1979),

${ }^{6}$ N. Nakanishi, Prog. Theor. Phys. 62, 1396 (1979).

${ }^{7}$ L. Bonora, P. Pasti, and M. Tonin, Nuovo Cimento A 64, 307 (1981).

${ }^{8}$ N. Nishijima, Nucl. Phys. B 238, 601 (1984).

${ }^{9}$ A. V. Razumov and G. N. Rybkin, Nucl. Phys. B 332, 209 (1990).

${ }^{10} \mathrm{~J}$. W. van Holten, Nucl. Phys. B 339, 158 (1990).

"B. L. Voronov and I. V. Tyutin, Theor. Math. Phys. 50, 218 (1982).

${ }^{12}$ I. A. Batalin and G. A. Vilkovisky, J. Math. Phys. 26, 172 (1985).

${ }^{13}$ D. M. Gitman and I. V. Tyutin, Quantization of fields with constraints, Springer Ser. Nucl. Part. Phys. (Springer, Berlin, 1990); I. A. Batalin, P. M. Lavrov, and I. V. Tyutin, J. Math. Phys. 31, 1487 (1990). 
${ }^{14}$ M. Henneaux, Commun. Math.Phys. 140, 1 (1991).

${ }^{15}$ M. Dubois-Violette, M. Henneaux, M. Talon, and C. M. Viallet, Phys. Lett. B 267, 81 (1991).

${ }^{16}$ J. M. L. Fisch and M. Henneaux, Commun. Math. Phys. 128, 627 (1990). See also M. Henneaux, Lectures on the antifield-BRST formalism for gauge theories, Proceedings of the XX GIFT International Seminar on Theoretical Physics, North-Holland Delta Series (North-Holland, Amsterdam, 1990).

${ }^{17}$ I. A. Batalin and G. A. Vilkovisky, Nucl. Phys. B 234, 106 (1984). 